TecnoLógicas

ISSN-p 0123-7799

ISSN-e 2256-5337

Vol. 22 , No. 45 , pp. $155-171$

Mayo-agosto de 2019

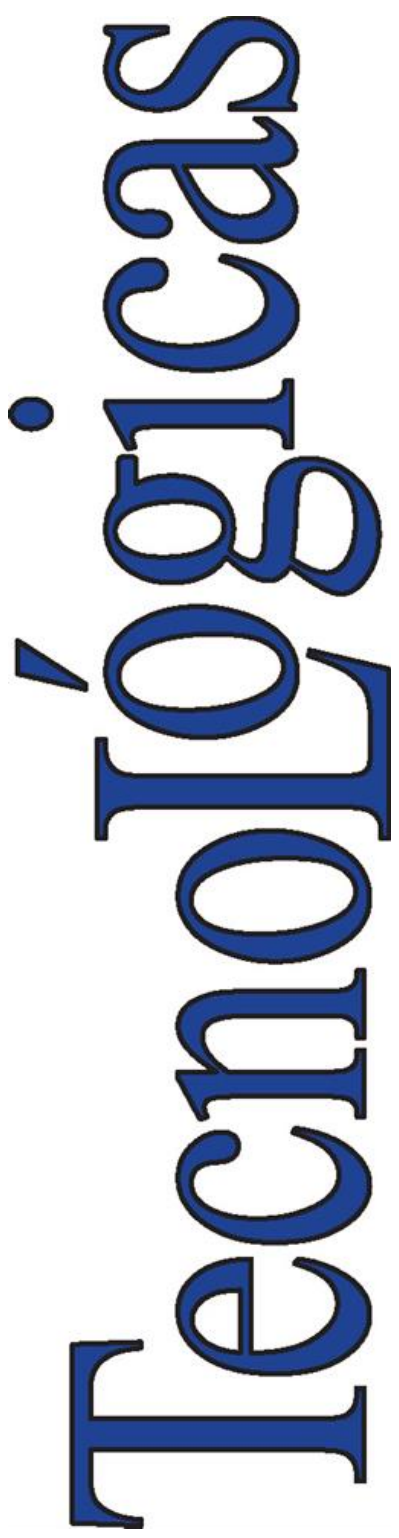

(C) Instituto Tecnológico Metropolitano Este trabajo está licenciado bajo una Licencia Internacional Creative Commons Atribución (CC BY-NC-SA)

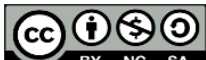

Artículo de Investigación/Research Article

\section{Comportamiento de descargadores de sobretensión ante tensiones inducidas por rayos sobre líneas de distribución ubicadas en zona montañosa}

\section{Behaviour of surge arresters against lightning-induced voltages on distribution lines over mountainous zones}

\author{
Edison Soto (D) ${ }^{1}$, Daniel Martínez (D)2, \\ y Diego Verdugo(D) ${ }^{3}$
}

Recibido: 30 de noviembre de 2018

Aceptado: 12 de abril de 2019

\section{Cómo citar / How to cite}

E. Soto, D. Martínez, D. Verdugo, "Comportamiento de descargadores de sobretensión ante tensiones inducidas por rayos sobre líneas de distribución ubicadas en zona montañosa”. TecnoLógicas, vol. 22, no. 45, pp. 155-171, 2019. https://doi.org/10.22430/22565337.1161

$1 \mathrm{PhD}$ en Ingeniería, Escuela de Ingeniería Eléctrica, Electrónica y de Telecomunicaciones, Universidad Industrial de Santander, BucaramangaColombia, easotor@uis.edu.co

2 Ingeniero Eléctrico, Escuela de Ingeniería Eléctrica, Electrónica y de Telecomunicaciones, Universidad Industrial de Santander, BucaramangaColombia, daalmago21@gmail.com

3 Ingeniero Eléctrico, Escuela de Ingeniería Eléctrica, Electrónica y de Telecomunicaciones, Universidad Industrial de Santander, BucaramangaColombia, diegover92@hotmail.com 


\section{Resumen}

Este artículo analiza cómo la ubicación de descargadores de sobretensión, en líneas de distribución sobre zonas montañosas, afecta las tensiones inducidas que se presentan en estas líneas a causa de rayos. Se consideraron cuatro configuraciones de terreno no plano, que son representativas de la topografía colombiana. La inclusión del terreno, el canal de la descarga, la línea de distribución y el cálculo de las tensiones inducidas, se realizó por medio del método de diferencias finitas en el dominio del tiempo en coordenadas cartesianas en tres dimensiones. Se encontró que las tensiones inducidas en terrenos no planos están entre 2 a 5 veces las que se obtienen para líneas sobre terrenos planos. En el caso de los terrenos A, C y D simulados, las tensiones inducidas superaron los umbrales de operación normales de los descargadores de sobretensión, lo que supone que aquellos ubicados en líneas de distribución en zonas de montaña tienen esfuerzos adicionales a los que se presentan en terreno plano. Sin embargo, hay una configuración (la B), donde no se superan dichos umbrales.

\section{Palabras clave}

Tensiones inducidas por rayo, terrenos no planos, descargadores de sobretensión, método de diferencias finitas en el dominio del tiempo, líneas de distribución.

\section{Abstract}

This paper analyzes how the location of surge arresters on distribution lines over mountainous zones affect the lightning-induced voltages in this kind of lines. Four configurations of non-flat terrains representative of the Colombian topography were chosen. The inclusion of the terrain, the return stroke channel, the distribution line and the calculation of lightning-induced voltages were done by means of the finite difference time domain method in Cartesian coordinates in three dimensions (3D-FDTD). It was found that the induced voltages in non-flat terrains are between 2 and 5 times the obtained for flat terrain. For the case of the simulated terrains A, C and D, the induced voltages surpass the normal operating thresholds of the surge arresters. It supposes that surge arresters placed on distribution lines in mountainous zones have additional strengths to those existing in flat terrains. Nevertheless, there is a configuration (B) where the threshold values are not exceeded.

\section{Keywords}

Lightning-induced voltages, non-flat terrains, surge arresters, Finite difference time domain method (FDTD), distribution lines. 
Comportamiento de descargadores de sobretensión ante tensiones inducidas por rayos sobre líneas de distribución ubicadas en zona montañosa

\section{INTRODUCCION}

El efecto de los impactos indirectos de rayos sobre líneas de distribución ha sido estudiado ampliamente en diferentes trabajos previamente [1][5], ya que son la principal causa de fallas en este tipo de líneas. Esto se debe al gran número de impactos cercanos a la red que generan inducciones de magnitud suficiente para producir flameos en la cadena de aisladores que por lo general tiene un bajo nivel de aislamiento [1]. El campo electromagnético producido por el rayo tiene un efecto significativo en el fenómeno de la tensión inducida [5][6]. Diferentes aspectos afectan la magnitud y la forma de onda del campo electromagnético. Recientemente, la topografía sobre la que se encuentra la línea ha entrado en consideración, teniendo en cuenta que muchos lugares del mundo se encuentran ubicados sobre zonas montañosas. Se ha encontrado, a través de simulaciones [7] y de experimentos [8], que existe un incremento del campo eléctrico producido por el rayo que repercute a su vez en que las tensiones inducidas sean mayores a las que se presentan en zonas de terreno plano [9] [11]. La protección de líneas de distribución con descargadores de sobretensión ante tensiones inducidas por rayos fue estudiada por Yokoyama [12], concluyendo que para una distancia de 200 $\mathrm{m}$ entre descargadores se obtiene la mejor protección. En [13] se analiza el efecto del espaciamiento entre dos descargadores de sobretensión (DPS) contiguos en la máxima tensión inducida a lo largo de la línea, concluyéndose que para separaciones de $200 \mathrm{~m}$ entre descargadores se obtiene el mejor desempeño. En [2] se calcula la tasa de fallas de una línea de distribución con descargadores ubicados cada 500 y cada 200 m, mostrándose nuevamente la efectividad para el último caso. Hasta ahora, en la literatura, no se han analizado aspectos relacionados con la protección de líneas de distribución en zona de montaña ante tensiones inducidas por rayos. Por tanto, en este trabajo, se estudia el comportamiento de descargadores de sobretensión puestos en los extremos de una línea de distribución típica ubicada sobre cuatro (4) zonas montañosas, ante las tensiones inducidas provocadas por impactos de rayos en algunas posiciones alrededor de la línea. Se presentarán conclusiones sobre el efecto del terreno en la magnitud de las tensiones inducidas, cómo influye la localización de los DPS en los extremos de la línea y cuál es la afectación de la línea de acuerdo con el lugar del impacto de la descarga. El artículo está organizado así: en la sección 2 se presenta la metodología para obtener las tensiones inducidas por rayos en redes ubicadas sobre zona de montaña, en la sección 3 y se listan los casos de simulación a ser analizados. En la sección 3 se exhiben los resultados y análisis y en la sección 4 se enuncian las conclusiones del trabajo.

\section{METOdología}

Si bien el campo electromagnético producido por rayo se ha calculado tradicionalmente a través de las ecuaciones de Master y Uman [14], en el problema a tratar, no es posible su uso ya que estas son solo válidas para terreno plano y al no existir hasta ahora fórmulas aplicables a terrenos irregulares, se recurre al uso de metodologías numéricas.

\subsection{Método FDTD}

Los campos eléctricos se calculan por medio del método de diferencias finitas en el dominio del tiempo en tres dimensiones (FDTD-3D) en coordenadas cartesianas según [15] [16]. Como un ejemplo, las ecuaciones (1), (2), (3) y (4) permiten calcular el campo eléctrico en la dirección $\mathrm{x}$ : 


$$
\begin{aligned}
E_{x}^{n}\left(i+\frac{1}{2}, j, k\right)= & K_{1} E_{x}^{n-1}\left(i+\frac{1}{2}, j, k\right) \\
& +K_{2}\left\{H_{z}^{n-\frac{1}{2}}\left(i+\frac{1}{2}, j+\frac{1}{2}, k\right)-H_{z}^{n-\frac{1}{2}}\left(i+\frac{1}{2}, j-\frac{1}{2}, k\right)-H_{y}^{n-\frac{1}{2}}\left(i+\frac{1}{2}, j, k+\frac{1}{2}\right)\right. \\
& \left.+H_{y}^{n-\frac{1}{2}}\left(i+\frac{1}{2}, j, k-\frac{1}{2}\right)\right\}
\end{aligned}
$$

Donde:

$$
\begin{gathered}
K_{1}=\frac{1-\frac{\sigma \Delta t}{2 \varepsilon}}{1+\frac{\sigma \Delta t}{2 \varepsilon}} \\
K_{2}=\frac{\Delta t}{\varepsilon \Delta s} \frac{1}{1+\frac{\sigma \Delta t}{2 \varepsilon}} \\
K_{3}=\frac{\Delta t}{\mu \Delta s}
\end{gathered}
$$

siendo:

Hy y $\mathrm{Hz}$ los campos magnéticos en las direcciones respectivas,

$\sigma$ y $\varepsilon$, la conductividad y permitividad del terreno respectivamente.

$\Delta t$ es el paso de tiempo.

$\Delta s$ es el paso de espacio.

$\mathrm{i}, \mathrm{j} \mathrm{y} \mathrm{k}$ son variables que indican las posiciones discretas en el espacio.

$\mathrm{n}$ indica la posición discreta en el tiempo.

El canal de la descarga del rayo se simula como arreglos de fuentes de corriente, que se encienden a medida que la descarga de retorno asciende por él, mediante un modelo TL. La corriente en la base del canal se asumió como una onda heidler [17] con un valor pico de $12 \mathrm{kA}$ y una máxima tasa de ascenso de $40 \mathrm{kA} / \mu \mathrm{s}$. El terreno por su parte se discretizó a través de cubos.

La línea de distribución se modeló como un conductor delgado variando en la dirección $\mathrm{x}$, siguiendo la metodología planteada en [18]. Se utilizó un paso de espacio $\Delta s$ de $5 \mathrm{~m}$ (en todas las direcciones) debido al gran volumen computacional requerido para un paso de espacio menor, sin embargo, es suficiente para tener errores por debajo del $2 \%$, en comparación con simulaciones realizadas usando un paso de espacio $\Delta s$ de $2.5 \mathrm{~m}$. Se utilizó un volumen con dimensiones 1200x500x980 $\mathrm{m}^{3}$ y con el fin de limitar el espacio computacional, todas las superficies cúbicas se trataron como fronteras absorbentes de Liao de segundo orden [19].

\subsection{Inclusión de los descargadores de sobretensión (DPS)}

La representación de los descargadores de sobretensión en el algoritmo FDTD 3D, se hace mediante las técnicas para la incorporación de dispositivos no lineales, tales como los diodos [16]. La curva característica (V-I) del DPS obtenida de las hojas de datos del fabricante, se puede discretizar como se muestra en la Fig.1 [20], siendo $I_{k}$ y $V_{k}$ la corriente y la tensión, respectivamente, en el punto k-ésimo especificado, y el número total de puntos es denotado por K. Se debe cumplir con las condiciones $V_{k}<V_{k+1}$ e $I_{k}<I_{k+1}$ [20].

La ecuación para actualizar el campo eléctrico situado alrededor del dispositivo no lineal está dada por las ecuaciones (5), (6) y (7): 
$\mathrm{E}^{\mathrm{n}+1}=\frac{1-\mathrm{C}}{1+\mathrm{C}} \mathrm{E}^{\mathrm{n}}+\frac{\Delta \mathrm{t}}{(1+\mathrm{C}) \varepsilon}(\nabla \mathrm{xH})$

Donde:

$$
\begin{gathered}
\mathrm{C}=\frac{\Delta \mathrm{t}}{2 * \mathrm{R}_{\mathrm{k} *} \varepsilon * \Delta_{\mathrm{s}}} \\
\mathrm{y} \\
\mathrm{R}_{\mathrm{k}}=\frac{V_{k+1}-V_{k}}{I_{k+1}-I_{k}}
\end{gathered}
$$

\subsection{Casos propuestos}

En esta sección se presenta la simulación de topografías típicas encontradas en Colombia en zona de montaña, de acuerdo con la revisión hecha en [10]. Los terrenos propuestos son:

-Terreno A: terreno en forma de V (Fig. 5)
-Terreno B: terreno en forma de V invertida. (Fig.8)

-Terreno C: terreno hueco (Fig.9)

-Terreno D: terreno en forma de W. Fig.10

Para cada terreno se varía su ángulo de inclinación entre $10^{\circ}, 20^{\circ}$ y $30^{\circ}$, siendo estos, valores típicos de terrenos encontrados [10].

Para cada una de las configuraciones anteriores, se simulan impactos en diferentes posiciones, como se muestra en la Fig.2:

-(A1) A 50 m del inicio de la línea

-(A2) A $50 \mathrm{~m}$ en frente de la línea y a $50 \mathrm{~m}$ del extremo izquierdo.

-(A3) A 50 m de la mitad de la línea.

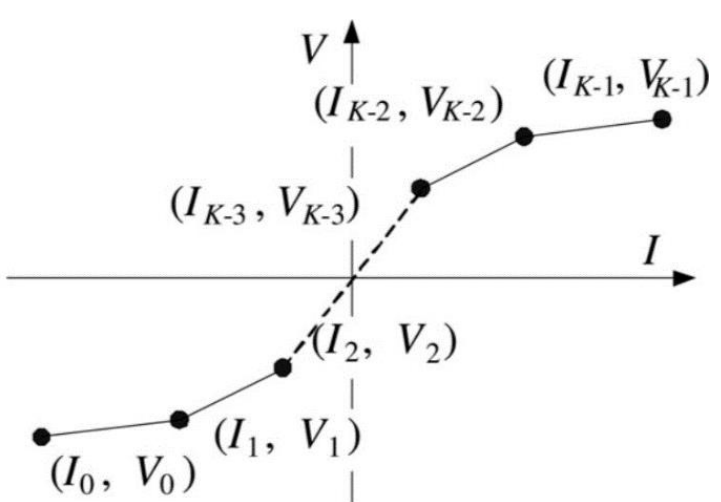

Fig. 1. Representación de la característica V-I del descargador. Fuente: [20].

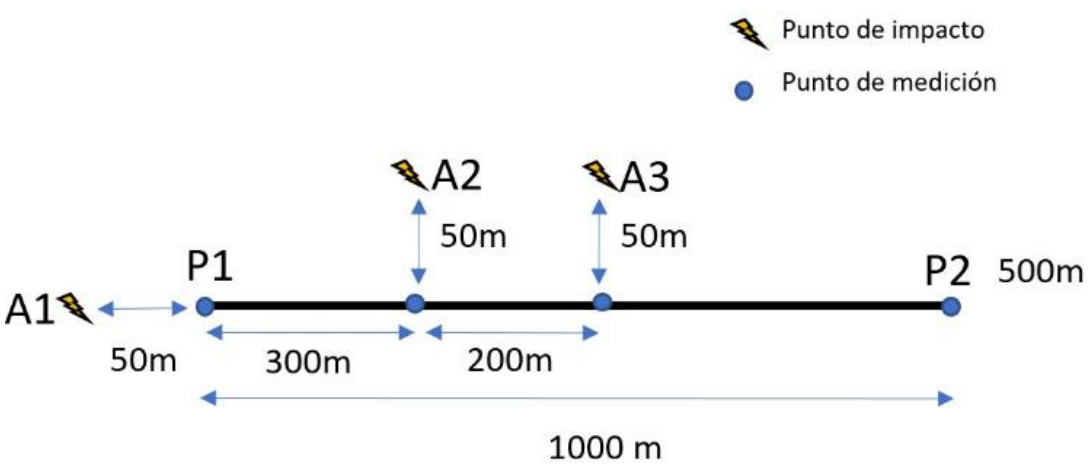

Fig. 2. Posiciones de impacto y de observación sobre la línea. Fuente: autores. 
A su vez se plantean diferentes posiciones de los DPS en los extremos de la línea:

\section{-Sin DPS}

-DPS al inicio de la línea (En P1)

-DPS al final de la línea (en P2)

-DPS al inicio y final de la línea (en P1 y 2, respectivamente)

Los valores característicos del DPS simulado para un nivel de tensión de 34,5 $\mathrm{kV}$ se presentan en la Fig. 3 el cual soporta una tensión máxima de $95,4 \mathrm{kV}$ para su nivel para la corriente nominal de descarga
[21] [22]. Adicionalmente en la Fig.4 se presenta la curva característica del DPS, con base en presentada en [20].

\section{RESULTADOS}

Aquí se presentan las simulaciones de los casos propuestos en la sección 2.3, se muestran los resultados de las tensiones inducidas para cada uno de los terrenos expuestos, para los tres puntos de impacto (A1, A2 y A3) y para las cuatro ubicaciones de DPS.

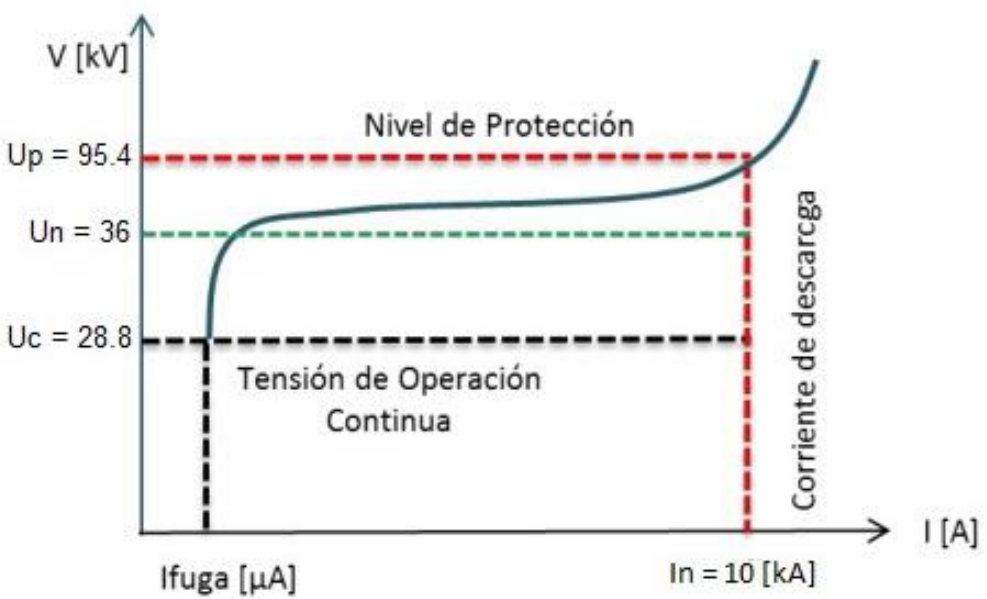

Fig. 3. Curva característica (V-I) de un DPS. Fuente: adaptado de [22].

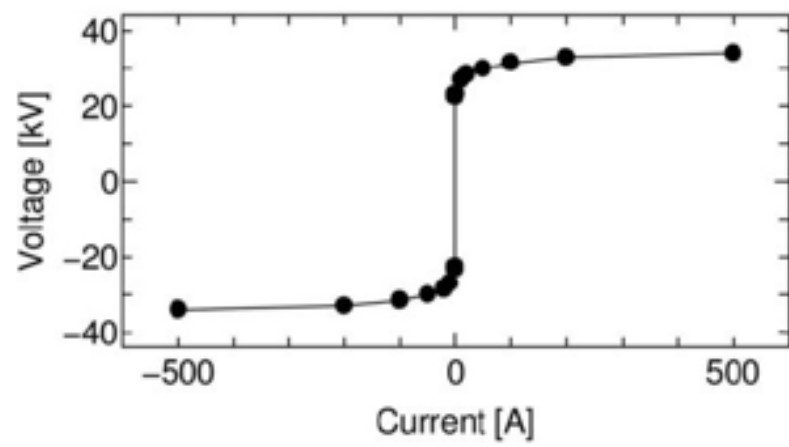

Fig. 4. Curva característica V-I del descargador empleado.

Fuente: [20]. 
Comportamiento de descargadores de sobretensión ante tensiones inducidas por rayos sobre líneas de distribución ubicadas en zona montañosa

\subsection{Resultados terreno $A$}

En esta sección se plantea la configuración del terreno A (terreno en forma de V) (ver Fig. 5). Este presenta una longitud de $1000 \mathrm{~m}$ y un ancho de $250 \mathrm{~m}$, $\mathrm{su}$ profundidad varía dependiendo del ángulo de inclinación $\alpha$.

Inicialmente se analizan las tensiones inducidas que resultan en los dos extremos de la línea (puntos de observación P1 y P2 en la Fig. 2), debido al impacto A1, cuando se varía la ubicación de los DPS. Así, la Fig. 6 corresponde al caso sin DPS, la Fig. 7 a los casos con DPS al inicio de la línea y con DPS al final de la línea. Es posible ver que la presencia del DPS disminuye a un valor cercano a $30 \mathrm{kV}$ la tensión inducida en cada nodo.

En la Tabla 1 se presentan los resultados obtenidos de la tensión inducida para la configuración de terreno A, considerando la inclinación del terreno $\left(10^{\circ}\right.$ - 30), la posición de la descarga (A1-A3) y la ubicación del DPS. Se puede resaltar que, al variar el ángulo de inclinación $\alpha$, considerando inicialmente la posición A1, la tensión inducida al inicio de la línea se incrementa levemente (cercana al $10 \%$ ) y al final de la línea disminuye en una misma proporción respecto al valor de tensión del ángulo anterior. Para el impacto A2, aumentar el ángulo equivale a incrementar la tensión inducida en ambos extremos de la línea. Para el impacto A3, no hay variación de la tensión inducida con el ángulo.

Observando los datos de la Tabla 1, se debe resaltar que en la configuración de terreno A, respecto a la configuración de terreno plano (Tabla 5), sin ubicar DPS, la tensión inducida aumenta en el inicio de la línea, en un $21 \%$ para inclinación de $10^{\circ}$, $38 \%$ para $20^{\circ}$ y $48 \%$ para $30^{\circ}$.

La presencia del DPS en alguno de los extremos de la línea lleva a que la tensión inducida disminuya a los valores de operación nominales del DPS (aproximadamente $\quad 30-36 \quad \mathrm{kV}$ ), especialmente para el impacto A1. Para los demás impactos, la tensión inducida en el extremo con DPS supera su límite de operación $(95 \mathrm{kV})$. Estos resultados se presentan como asteriscos al salirse de los valores que podría predecir el modelo del descargador. En estas condiciones, un descargador sobre una línea que se encuentre ubicada en una topografía como la del terreno en A funcionaría eficazmente ante el impacto A1, pero no para A2 y A3.

La explicación de los resultados anteriores viene de analizar la existencia de tres factores que afectan la magnitud de la tensión inducida: la inclinación del terreno, la posición de la descarga y la ubicación del DPS. En el primer aspecto, según se ha mostrado en trabajos previos [7][11], la pendiente del terreno juega un papel importante en la magnitud del campo eléctrico vertical y horizontal que se presenta a la altura de la línea. La inducción de estos campos con la línea hace que la tensión inducida también sea mayor cuando se compara con casos de terrenos planos. En el segundo aspecto, el ángulo de incidencia de la descarga respecto a la línea influye en la tensión inducida. Por ejemplo, en la posición A3, la descarga está ubicada justo en frente de la mitad de la línea y en este punto es donde se presenta la mayor inducción, por esta razón la tensión inducida es superior a todos los demás casos. Por último, la ubicación del DPS, determina la reducción de la tensión inducida en el sitio donde se ubique.

\subsection{Resultados terreno B}

En esta sección se plantea la configuración de un terreno en forma de $\mathrm{V}$ invertida (ver Fig.8). Posee una longitud de $1200 \mathrm{~m}$, un ancho de $500 \mathrm{~m}$ y su altura varía de acuerdo con el ángulo de inclinación $\alpha$. La línea se ubica en la parte más elevada del terreno, el cual tiene un ancho de $100 \mathrm{~m}$. 
Comportamiento de descargadores de sobretensión ante tensiones inducidas por rayos sobre líneas de distribución ubicadas en zona montañosa

a)
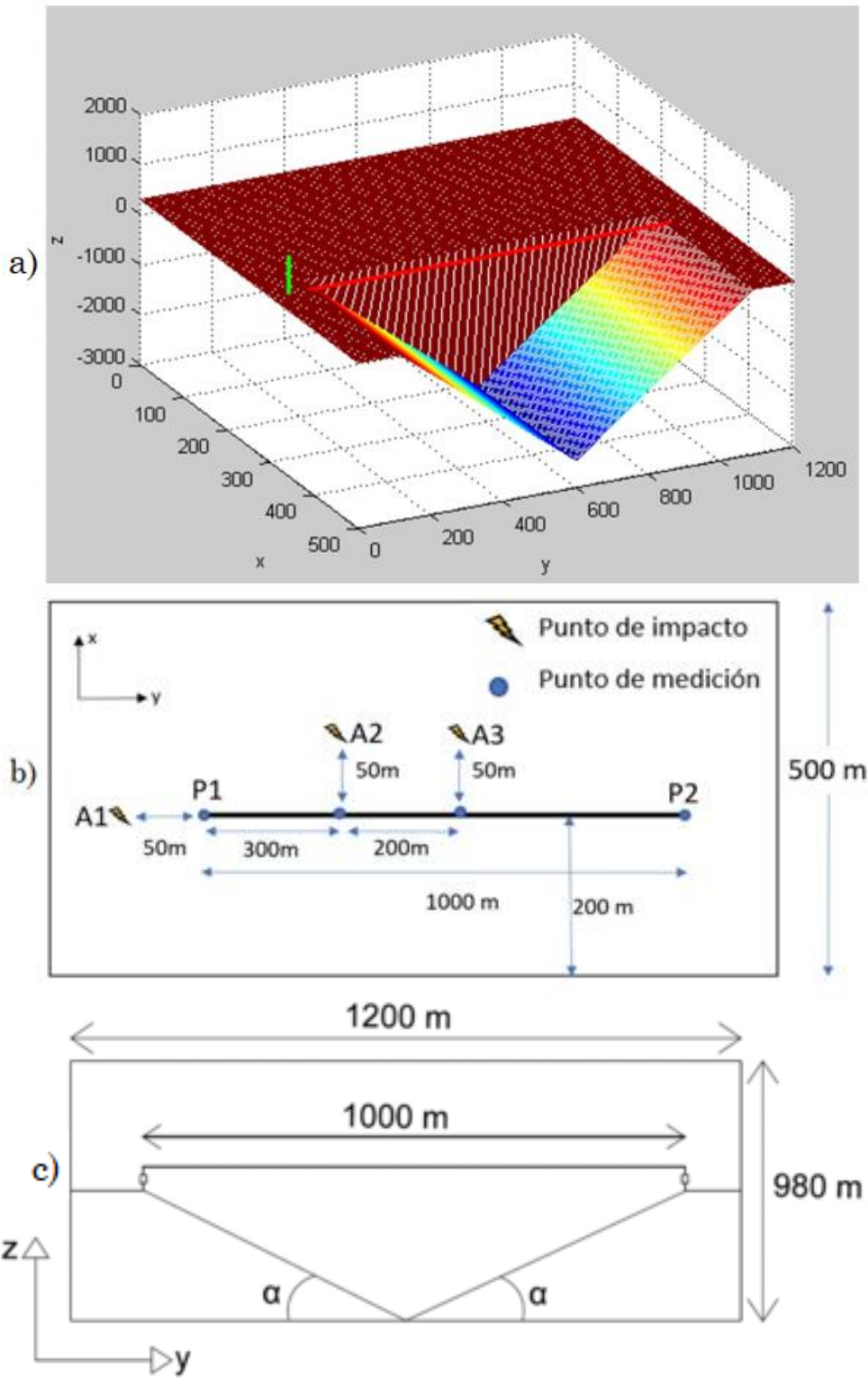

Fig. 5. Vista 3D (a), vista superior (b) y vista frontal (c) del terreno A (terreno en forma de V).

Fuente: autores.

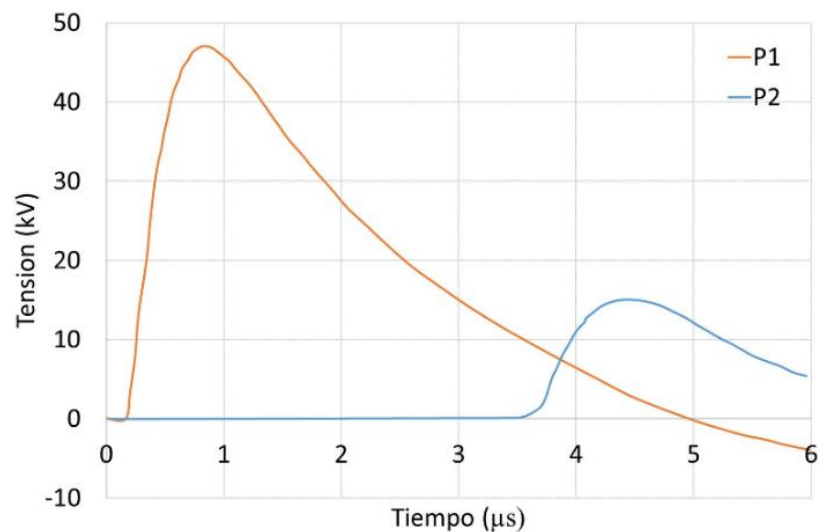

Fig. 6. Tensión inducida para el caso sin DPS, al inicio (P1) y al final de la línea (P2) para el terreno A. Fuente: autores. 
Comportamiento de descargadores de sobretensión ante tensiones inducidas por rayos sobre líneas de distribución ubicadas en zona montañosa

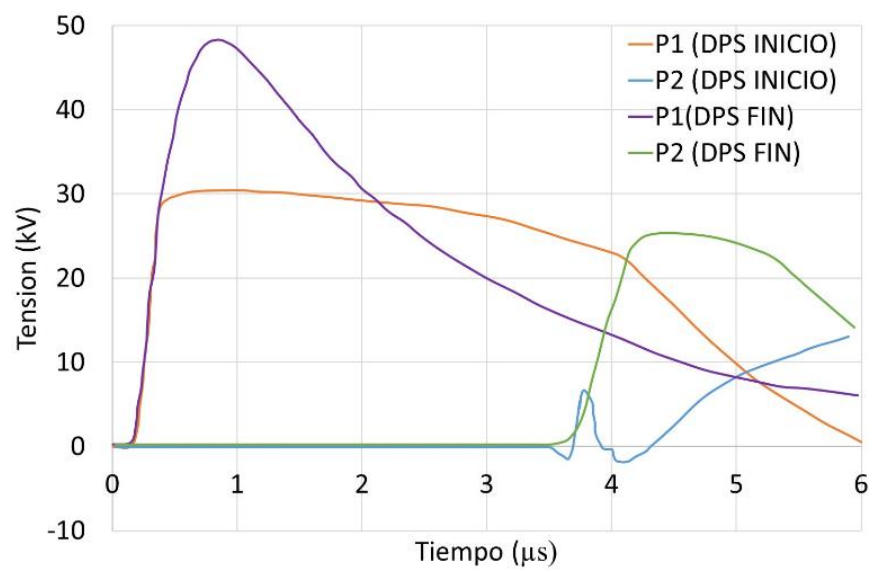

Fig. 7. Tensión inducida para el caso con DPS al inicio de la línea y DPS al final de la línea, en los puntos (P1) y (P2) para el terreno A. Fuente: autores.

a)
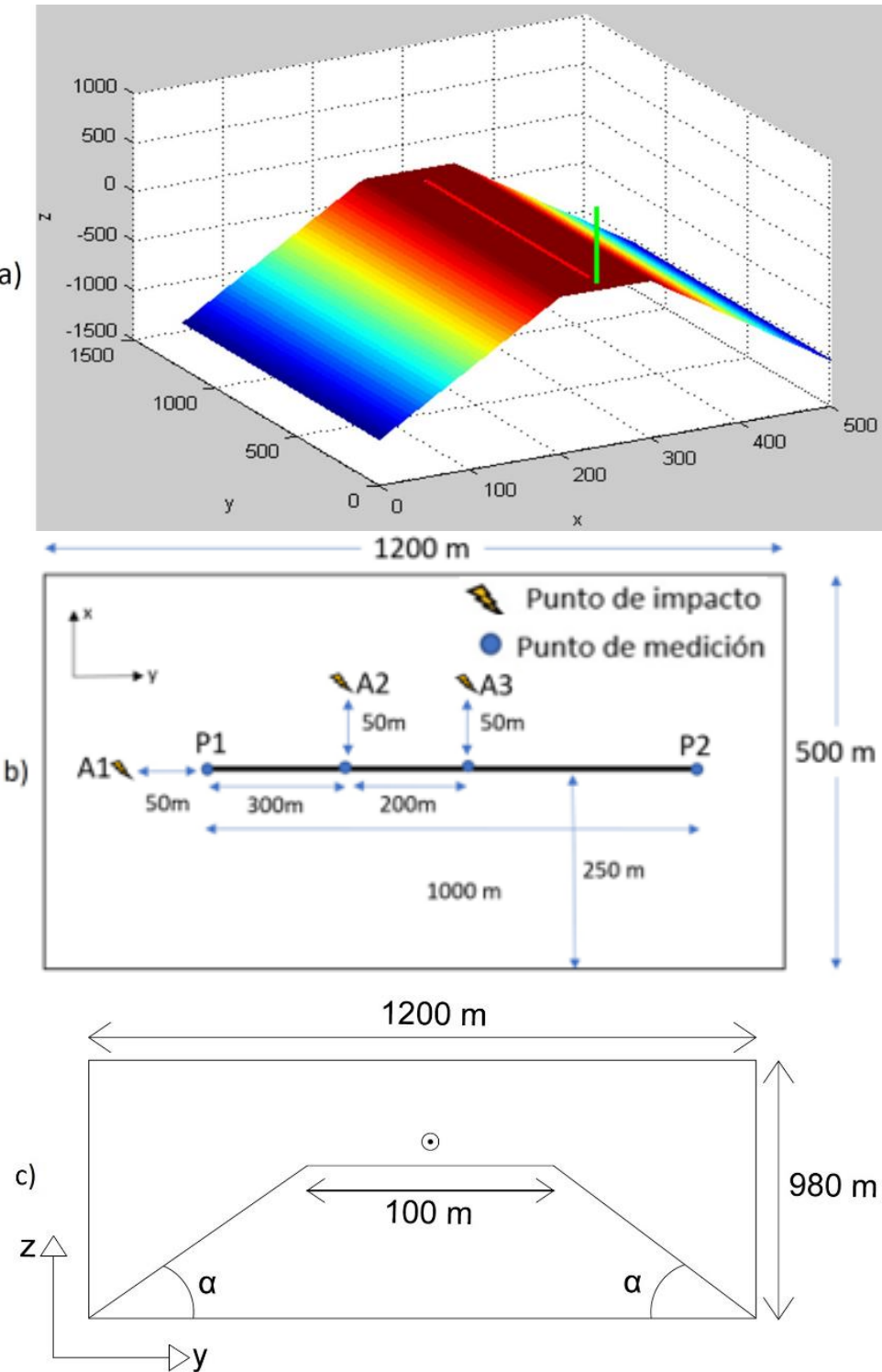

Fig. 8. Vista 3D (a), vista superior (b) y vista frontal (c) del terreno B (terreno de montaña). Fuente: autores. 
Haciendo un estudio similar al presentado en el caso anterior, se llega a los resultados presentados en la Tabla 1 . En esta se puede resaltar que, al variar el ángulo de inclinación del terreno, la tensión inducida al inicio de la línea cuando no hay presencia del descargador de sobretensión, se incrementa aproximadamente en un $2 \%$ respecto al valor de tensión del ángulo anterior y al final de la línea no presenta cambio; por lo tanto, el incremento en la tensión que se presenta por la variación del ángulo no es significativo en el terreno montañoso.

Cuando no hay presencia del DPS, se debe resaltar que en la configuración de terreno $\mathrm{B}$, respecto a la configuración de terreno plano (Tabla 5), la tensión inducida aumenta en el inicio de la línea, en un $1 \%$ para inclinación de $10^{\circ}, 3 \%$ para $20^{\circ}$ y $5 \%$ para $30^{\circ}$. Lo cual confirma la observación anterior.
En la Tabla 2, cuando se ubica el DPS al inicio de la línea, y se analiza el impacto A1, se puede apreciar que además de reducir los valores de tensión en su nodo de aplicación, también se incrementan de manera significativa las tensiones al final de la línea aproximadamente 1,4 veces el valor sin DPS. Para los demás impactos (A2 y A3), la presencia del descargador en uno de los extremos, permanece invariable la sobretensión que se presentaba cuando no había descargadores.

Cabe resaltar que, para la configuración de terreno $\mathrm{B}$, al cambiar el punto de impacto de la descarga (A2 o A3) se observa que los resultados están en el rango de acción del DPS $(95,4 \mathrm{kV})$, lo que quiere decir que el descargador utilizado es el indicado para la protección de líneas de distribución en este tipo de configuración.

Tabla 1. Máxima tensión inducida en kV para la configuración del terreno A. Fuente: autores.

\begin{tabular}{|c|c|c|c|c|c|c|c|}
\hline \multirow[b]{2}{*}{$\begin{array}{l}\text { Punto } \\
\text { de } \\
\text { impacto }\end{array}$} & \multirow{2}{*}{$\begin{array}{l}\text { Inclinación } \\
(\alpha) \\
\text { Ubicación del } \\
\text { DPS }\end{array}$} & \multicolumn{2}{|r|}{$10^{\circ}$} & \multicolumn{2}{|c|}{$20^{\circ}$} & \multicolumn{2}{|c|}{$30^{\circ}$} \\
\hline & & $\begin{array}{l}\text { Inicio de } \\
\text { línea }(\mathrm{P} 1)\end{array}$ & $\begin{array}{l}\text { Final de } \\
\text { línea }(\mathrm{P} 2)\end{array}$ & $\begin{array}{l}\text { Inicio de } \\
\text { línea (P1) }\end{array}$ & $\begin{array}{l}\text { Final de } \\
\text { línea (P2) }\end{array}$ & $\begin{array}{l}\text { Inicio de } \\
\text { línea (P1) }\end{array}$ & $\begin{array}{l}\text { Final de } \\
\text { línea }(\mathrm{P} 2)\end{array}$ \\
\hline \multirow{4}{*}{$\mathrm{A} 1$} & Sin DPS & 46,9 & 15 & 53,4 & 13,1 & 57,3 & 11 \\
\hline & DPS inicio & 30,5 & 14 & 30,9 & 11,3 & 31,1 & 10,4 \\
\hline & DPS final & 46,9 & 25,4 & 53,4 & 24,2 & 30,9 & 22,1 \\
\hline & $\begin{array}{l}\text { DPS inicio y } \\
\text { final }\end{array}$ & 30,5 & 24,8 & 30,5 & 24,8 & 31,1 & 19 \\
\hline \multirow{4}{*}{$\mathrm{A} 2$} & Sin DPS & 128,5 & 134,1 & 136,4 & 150,1 & 138,3 & 155,3 \\
\hline & DPS inicio & $* *$ & 134,1 & $* *$ & 150,1 & $* *$ & 155,3 \\
\hline & DPS final & 128,5 & $* *$ & 136,4 & $* *$ & 138,4 & $* *$ \\
\hline & $\begin{array}{l}\text { DPS inicio y } \\
\text { final }\end{array}$ & $* *$ & $* *$ & $* *$ & $* *$ & $* *$ & $* *$ \\
\hline \multirow{4}{*}{ A3 } & Sin DPS & 147,9 & 147,9 & 151,5 & 151,5 & 151,9 & 151,9 \\
\hline & DPS inicio & $* *$ & 147,9 & $* *$ & 151,5 & $* *$ & 151,9 \\
\hline & DPS final & 147,9 & $* *$ & 151,5 & $* *$ & 151,9 & $* *$ \\
\hline & $\begin{array}{l}\text { DPS inicio y } \\
\text { final }\end{array}$ & $* *$ & $* *$ & $* *$ & $* *$ & $* *$ & $* *$ \\
\hline
\end{tabular}

**Fuera de rango 
Comportamiento de descargadores de sobretensión ante tensiones inducidas por rayos sobre líneas de distribución ubicadas en zona montañosa

\subsection{Resultados terreno C}

En esta sección se plantea la configuración del terreno $\mathrm{C}$ (terreno hueco Fig.9). Su parte hueca presenta un ancho de $100 \mathrm{~m}$, una longitud de $1000 \mathrm{~m}$ y la profundidad del hueco varía con la inclinación del ángulo. Su forma presenta similitudes con el terreno A (terreno en V). La línea se ubica por encima del hueco de manera centrada.
Para esta sección se presentan los resultados de las tensiones máximas tabuladas de la misma manera como se hizo en la sección anterior (ver Tabla 3).

Se puede observar, al igual que para los resultados del terreno $\mathrm{A}$, que, variando el ángulo de inclinación del terreno, la tensión inducida al inicio de la línea cuando no hay presencia del descargador de sobretensión se incrementa levemente respecto al valor de tensión del ángulo anterior.

a)
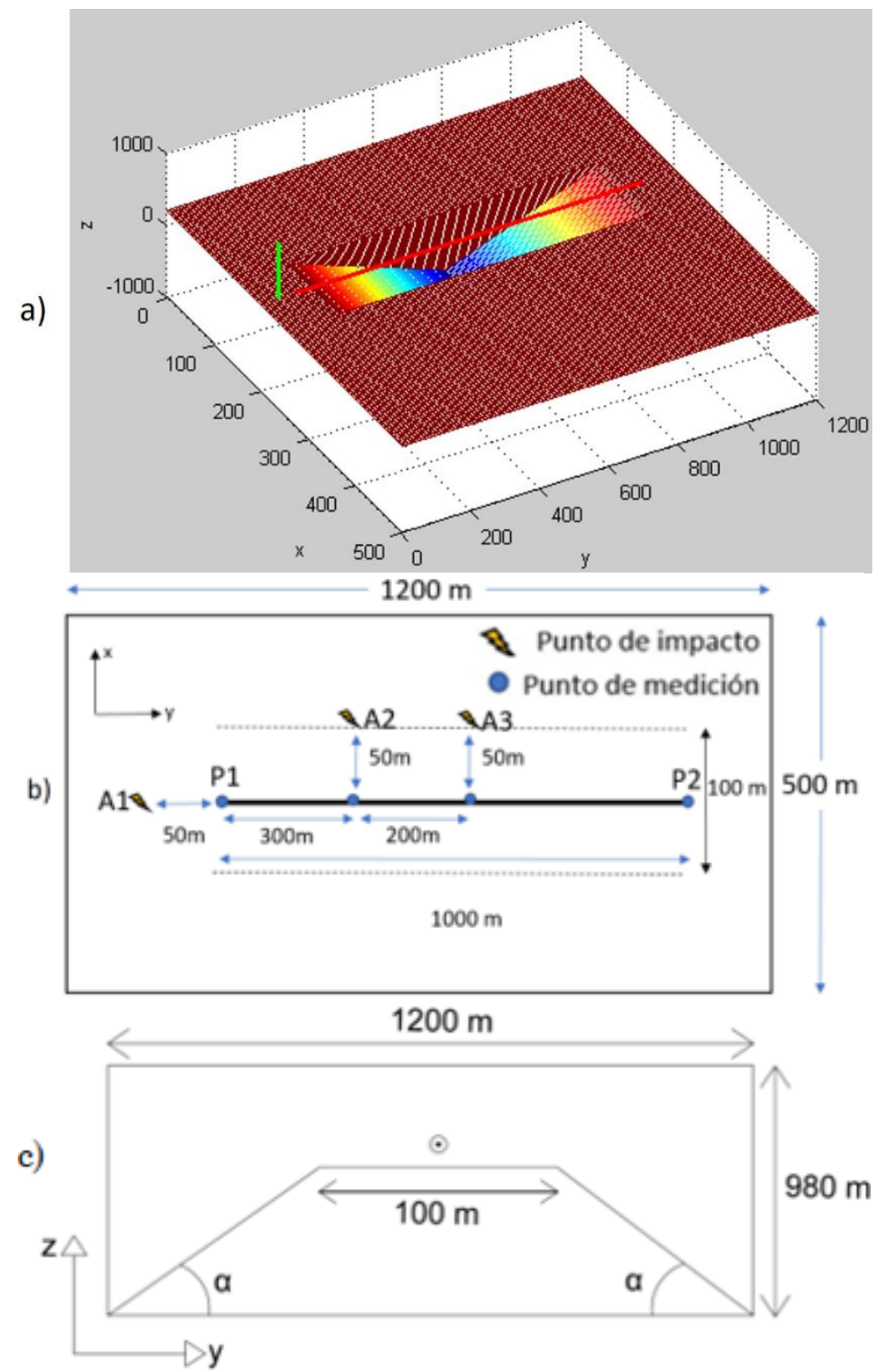

Fig. 9. Vista 3D (a), superior (b) y frontal (c) del terreno C (terreno hueco). Fuente: autores. 
Comportamiento de descargadores de sobretensión ante tensiones inducidas por rayos sobre líneas de distribución ubicadas en zona montañosa

Los resultados de tensión inducida del terreno $\mathrm{C}$ respecto al terreno plano (Tabla 5) cuando no hay presencia del DPS y se considera el impacto $\mathrm{A} 1$, presentan un aumento en el inicio de la línea, en un $3 \%$ para inclinación de $10^{\circ}, 12 \%$ para $20^{\circ}$ y 19 $\%$ para $30^{\circ}$. En la Tabla 3, se puede apreciar que el DPS actúa de la forma esperada (disminuyendo el valor de la tensión a un valor aproximado de $30 \mathrm{kV}$ ) en todos los casos del impacto A1, pero no lo hace para los demás. Por ejemplo, para los impactos A2 y A3, ubicar DPS en ambos extremos de la línea resulta en algo negativo porque ambos descargadores fallarán.

Tabla 2. Máxima tensión inducida en $\mathrm{kV}$ para la configuración del terreno B. Fuente: autores.

\begin{tabular}{|c|c|c|c|c|c|c|c|}
\hline & Inclinación $(\alpha)$ & & $10^{\circ}$ & & $20^{\circ}$ & & $30^{\circ}$ \\
\hline $\begin{array}{l}\text { Punto } \\
\text { de } \\
\text { impacto }\end{array}$ & $\begin{array}{l}\text { Ubicación del } \\
\text { DPS }\end{array}$ & $\begin{array}{l}\text { Inicio de } \\
\text { línea (P1) }\end{array}$ & $\begin{array}{c}\text { Final de } \\
\text { línea (P2) }\end{array}$ & $\begin{array}{l}\text { Inicio de } \\
\text { línea (P1) }\end{array}$ & $\begin{array}{c}\text { Final de } \\
\text { línea (P2) }\end{array}$ & $\begin{array}{l}\text { Inicio de } \\
\text { línea (P1) }\end{array}$ & $\begin{array}{l}\text { Final de } \\
\text { línea (P2) }\end{array}$ \\
\hline \multirow{4}{*}{ A1 } & Sin DPS & 39,3 & 14,1 & 40,1 & 14,4 & 40,6 & 14,8 \\
\hline & DPS inicio & 30,2 & 20,6 & 30,3 & 20,6 & 30,4 & 21,1 \\
\hline & DPS final & 39,2 & 24,9 & 40,1 & 25,1 & 40,6 & 25,3 \\
\hline & DPS inicio y final & 30,1 & 27,5 & 30,3 & 27,5 & 30,2 & 27,5 \\
\hline \multirow{4}{*}{$\mathrm{A} 2$} & Sin DPS & 66,8 & 68,4 & 68,7 & 70,3 & 70,1 & 71,6 \\
\hline & DPS inicio & 30,6 & 68,4 & 30,7 & 70,3 & 30,8 & 71,6 \\
\hline & DPS final & 66,8 & 31,3 & 68,7 & 31,4 & 70,1 & 31,5 \\
\hline & DPS inicio y final & 30,6 & 31,3 & 30,7 & 31,4 & 30,8 & 31,5 \\
\hline \multirow{4}{*}{ A3 } & Sin DPS & 67,9 & 67,9 & 69,9 & 69,9 & 71,4 & 71,4 \\
\hline & DPS inicio & 31 & 67,9 & 31,1 & 69,9 & 31,2 & 71,4 \\
\hline & DPS final & 67,9 & 31,3 & 69,9 & 31,4 & 71,4 & 31,5 \\
\hline & DPS inicio y final & 31,3 & 31,3 & 31,4 & 31,4 & 31,5 & 31,5 \\
\hline
\end{tabular}

Tabla 3. Máxima tensión inducida para la configuración del terreno en C. Fuente: autores.

\begin{tabular}{|c|c|c|c|c|c|c|c|}
\hline \multirow[b]{2}{*}{$\begin{array}{l}\text { Punto de } \\
\text { impacto }\end{array}$} & Inclinación $(\alpha)$ & \multicolumn{2}{|r|}{$10^{\circ}$} & \multicolumn{2}{|r|}{$20^{\circ}$} & \multicolumn{2}{|r|}{$30^{\circ}$} \\
\hline & $\begin{array}{l}\text { Ubicación del } \\
\text { DPS }\end{array}$ & $\begin{array}{l}\text { Inicio de } \\
\text { línea }(\mathrm{P} 1)\end{array}$ & $\begin{array}{l}\text { Final de } \\
\text { línea }(\mathrm{P} 2)\end{array}$ & $\begin{array}{l}\text { Inicio de } \\
\text { línea }(\mathrm{P} 1)\end{array}$ & $\begin{array}{l}\text { Final de } \\
\text { línea }(\mathrm{P} 2)\end{array}$ & $\begin{array}{l}\text { Inicio de } \\
\text { línea }(\mathrm{P} 1)\end{array}$ & $\begin{array}{l}\text { Final de } \\
\text { línea (P2) }\end{array}$ \\
\hline \multirow[t]{4}{*}{ A1 } & Sin DPS & 39,7 & 14,9 & 43,6 & 14,8 & 46,4 & 14,8 \\
\hline & DPS inicio & 30,1 & 16,1 & 30,3 & 16,2 & 30,4 & 16,4 \\
\hline & DPS final & 39,7 & 25,3 & 43,6 & 25,3 & 46,4 & 25,41 \\
\hline & $\begin{array}{l}\text { DPS inicio } y \\
\text { final }\end{array}$ & 30 & 26,1 & 30,3 & 26,1 & 30,4 & 26,2 \\
\hline \multirow[t]{4}{*}{$\mathrm{A} 2$} & Sin DPS & 118,5 & 119,4 & 122,9 & 127,3 & 123,8 & 129,6 \\
\hline & DPS inicio & $* *$ & 119,4 & $* *$ & 127,3 & $* *$ & 129,6 \\
\hline & DPS final & 118,5 & $* *$ & 122,9 & $* *$ & 123,8 & $* *$ \\
\hline & $\begin{array}{l}\text { DPS inicio y } \\
\text { final }\end{array}$ & $* *$ & $* *$ & $* *$ & $* *$ & $* *$ & $* *$ \\
\hline \multirow[t]{4}{*}{ A3 } & Sin DPS & 129,7 & 129,7 & 130,2 & 130,2 & 130,2 & 130,2 \\
\hline & DPS inicio & $* *$ & 129,7 & $* *$ & 130,2 & $* *$ & 130,2 \\
\hline & DPS final & 129,7 & $* *$ & 130,2 & $* *$ & 130,3 & $* *$ \\
\hline & $\begin{array}{l}\text { DPS inicio } y \\
\text { final }\end{array}$ & $* *$ & $* *$ & $* *$ & $* *$ & $* *$ & $* *$ \\
\hline
\end{tabular}

$* *$ Fuera de rango

[166] TecnoLógicas, ISSN-p 0123-7799 / ISSN-e 2256-5337, Vol. 22, No. 45, mayo-agosto de 2019, pp. 155-171 


\subsection{Resultado terreno D}

En esta sección se plantea la configuración del terreno D (terreno en W). Esta es una configuración híbrida, la cual se propone de manera tal que incluya los rasgos más significativos de las demás configuraciones (ver Fig.10). El terreno presenta un ancho de $250 \mathrm{~m}$, una longitud de $1000 \mathrm{~m}$, en su parte hueca se presentan dos pendientes en forma de $\mathrm{V}$ ubicadas a lado y lado de manera simétrica, las cuales permanecen iguales así se varíe el ángulo de inclinación. En medio de estas pendientes se presenta una elevación simulando el terreno de montaña donde su parte más elevada presenta una planicie con un ancho de $100 \mathrm{~m}$ y su altura varía de acuerdo con el ángulo seleccionado $\alpha$.

En la Tabla 4, se presentan los resultados obtenidos de la tensión inducida para esta configuración, teniendo en cuenta cada posición del descargador de sobretensión y cada ángulo de inclinación del terreno. Se puede observar que para el impacto A1, al variar el ángulo de inclinación del terreno, la tensión inducida al inicio de la línea cuando no hay presencia del descargador de sobretensión se incrementa aproximadamente en un $6 \%$ y al final de la línea disminuye en una proporción de $13 \%$ respecto al valor de tensión del ángulo anterior. Para el caso de otros impactos esta variación puede ser mayor.

Los resultados de tensión inducida del terreno D respecto al terreno plano (Tabla 5) con la descarga a $50 \mathrm{~m}$ del inicio de la línea (A1) sin la presencia del DPS, presentan un aumento en el inicio de la línea, en un $2 \%$ para inclinación de $10^{\circ}, 8$ $\%$ para $20^{\circ}$ y $15 \%$ para $30^{\circ}$. Para el impacto A3, la variación puede ser de hasta 220 veces.

De igual manera que en el terreno $\mathrm{C}$, para el punto de impacto A2 y A3 los descargadores de sobretensión están más allá de sus límites operativos. El nodo en el que no se conecta el DPS mantiene su tensión inmodificada, respecto al caso base sin DPS.

Tabla 4. Máxima tensión inducida para la configuración del terreno en D. Fuente: autores.

\begin{tabular}{|c|c|c|c|c|c|c|c|}
\hline \multirow[b]{2}{*}{$\begin{array}{l}\text { Punto de } \\
\text { impacto }\end{array}$} & \multicolumn{2}{|l|}{ Inclinación ( $\alpha$ ) } & \multicolumn{2}{|l|}{$10^{\circ}$} & \multicolumn{2}{|l|}{$20^{\circ}$} & $30^{\circ}$ \\
\hline & $\begin{array}{l}\text { Ubicación del } \\
\text { DPS }\end{array}$ & $\begin{array}{l}\text { Inicio de } \\
\text { línea }(\mathrm{P} 1)\end{array}$ & $\begin{array}{l}\text { Final de } \\
\text { línea }(\mathrm{P} 2)\end{array}$ & $\begin{array}{l}\text { Inicio de } \\
\text { línea }(\mathrm{P} 1)\end{array}$ & $\begin{array}{l}\text { Final de } \\
\text { línea (P2) }\end{array}$ & $\begin{array}{l}\text { Inicio de } \\
\text { línea }(\mathrm{P} 1)\end{array}$ & $\begin{array}{l}\text { Final de } \\
\text { línea (P2) }\end{array}$ \\
\hline \multirow[t]{4}{*}{$\mathrm{A} 1$} & Sin DPS & 39,4 & 13,8 & 42 & 12,2 & 44,6 & 10 \\
\hline & DPS inicio & 30,1 & 14,1 & 30,3 & 11,7 & 30,4 & 11,2 \\
\hline & DPS final & 39,4 & 24,7 & 42 & 23,9 & 44,6 & 21,7 \\
\hline & $\begin{array}{l}\text { DPS inicio y } \\
\text { final }\end{array}$ & 30,1 & 25,1 & 30,3 & 23,7 & 30,4 & 22,3 \\
\hline \multirow[t]{4}{*}{$\mathrm{A} 2$} & Sin DPS & 124,1 & 124,7 & 132,9 & 141,3 & 135,3 & 148,4 \\
\hline & DPS inicio & $* *$ & 124,7 & $* *$ & 141,3 & $* *$ & 148,4 \\
\hline & DPS final & 124,1 & $* *$ & 132,9 & $* *$ & 135,3 & $* *$ \\
\hline & $\begin{array}{l}\text { DPS inicio y } \\
\text { final }\end{array}$ & $* *$ & $* *$ & $* *$ & $* *$ & $* *$ & $* *$ \\
\hline \multirow[t]{4}{*}{ A3 } & Sin DPS & 121,3 & 121,3 & 137,9 & 137,9 & 145 & 145 \\
\hline & DPS inicio & $* *$ & 121,3 & $* *$ & 137,9 & $* *$ & 145 \\
\hline & DPS final & 121,3 & $* *$ & 137,9 & $* *$ & 145 & $* *$ \\
\hline & $\begin{array}{l}\text { DPS inicio y } \\
\text { final }\end{array}$ & $* *$ & $* *$ & $* *$ & $* *$ & $* *$ & $* *$ \\
\hline
\end{tabular}

$* *$ Fuera de rango 
Comportamiento de descargadores de sobretensión ante tensiones inducidas por rayos sobre líneas de distribución ubicadas en zona montañosa

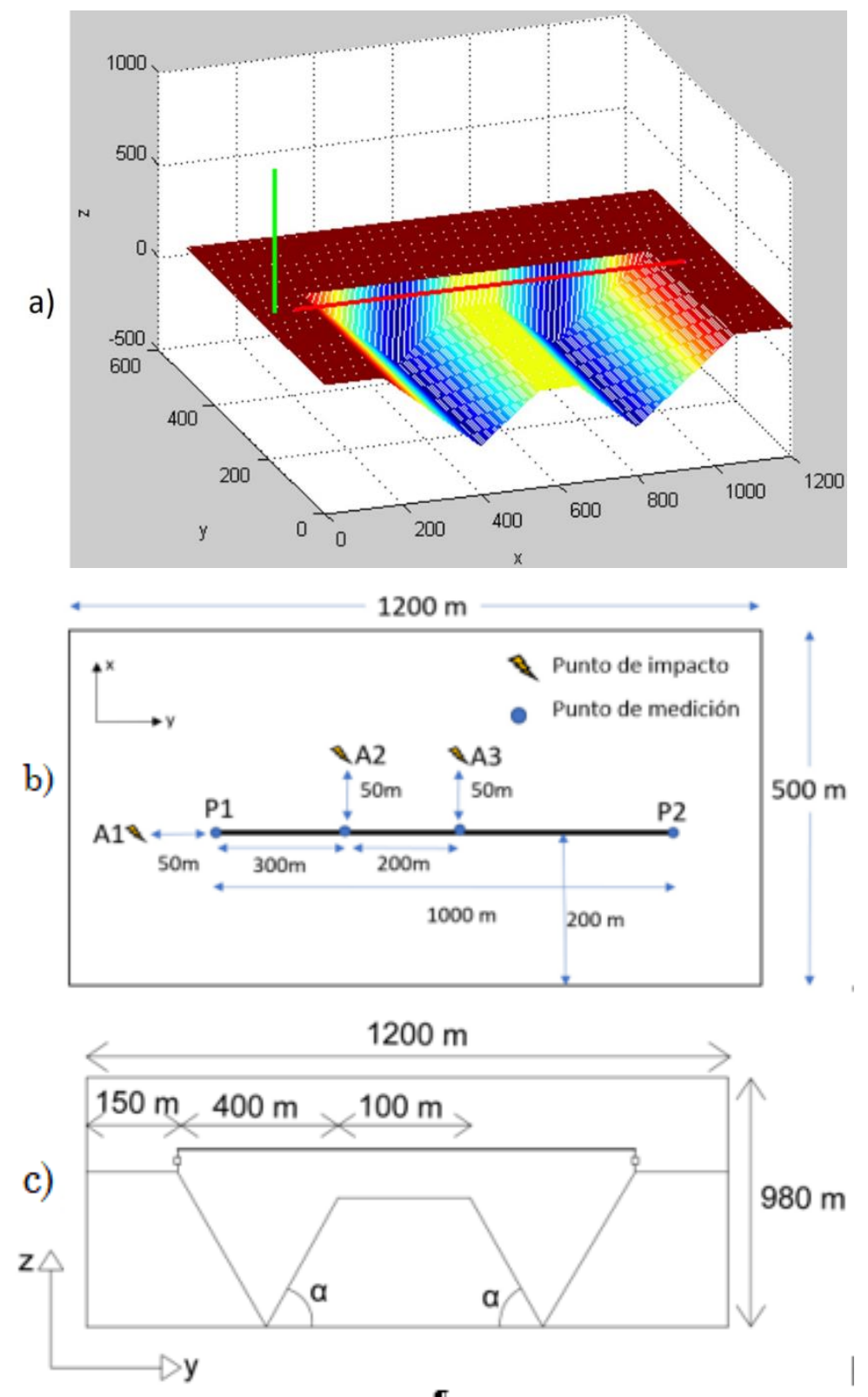

Fig. 10. Vista 3D (a), superior (b) y frontal (c) del terreno D (terreno en forma de W). Fuente: autores.

\section{ANÁLISIS DE RESULTADOS}

Teniendo en cuenta los resultados obtenidos de las diferentes configuraciones de terrenos propuestos, se concluye que el terreno A es el terreno más problemático al momento de instalar una línea de distribución, debido a que presenta los valores de tensión inducida más elevados de las cuatro configuraciones; y el terreno más apropiado para instalar la línea de distribución es el terreno $\mathrm{B}$, puesto que sus valores son muy similares a los de la configuración de terreno plano. Aunque es claro que una línea de distribución se instala en el lugar en el que se necesita, de acuerdo con el terreno, se deberán considerar especificaciones adicionales o no de los descargadores de sobretensión a instalar.

Al igual que en terreno plano, el descargador de sobretensión no siempre va a disminuir la tensión inducida. Se observa en todas las configuraciones de terreno no 
plano un aumento en la tensión inducida al final de la línea de 1,5 a 2 veces respecto al valor de tensión cuando no hay presencia del DPS, esto ocurre cuando la descarga es al inicio de la línea (A1) y el descargador está ubicado al final de la línea.

Para las configuraciones de terrenos A, C y D, los resultados de tensión obtenidos superan el rango máximo de operación del DPS $(95,4 \mathrm{kV})$, por lo tanto, el descargador utilizado no cumpliría con las exigencias necesarias para la protección de una línea de un sistema de distribución y se tendrían que implementar otros métodos adicionales de protección para dar mayor confiabilidad, seguridad y calidad al sistema de distribución.

\section{CONCLUSIONES}

En este artículo se ha calculado el efecto de la ubicación de descargadores de sobretensión sobre líneas ubicadas en zonas de montañosa ante impactos indirectos de rayos. Se ha podido comprobar que el efecto del terreno incrementa las tensiones inducidas entre 2 a 5 veces las que se presentan en terrenos planos. Hay configuraciones en las que las tensiones inducidas se incrementan menos, como es el caso de la configuración B.

En general, en el nodo donde se conecta el descargador, la tensión inducida cae a aproximadamente $30 \mathrm{kV}$. Sin embargo, los DPS no funcionan correctamente en los terrenos $\mathrm{A}, \mathrm{C}$ y $\mathrm{D}$, debido a que los valores obtenidos de tensión inducida están muy por encima del rango de operación (especialmente para los impactos A2 y A3), por lo que se producirán averías en el dispositivo. En estos casos, la alternativa sería utilizar descargadores para un nivel de tensión mayor.

Para mejorar la calidad de la energía y minimizar la salida de funcionamiento de las líneas, se debe tener en cuenta la configuración del terreno donde va a ser utilizado el DPS, puesto que los diferentes estudios para su selección están desarrollados en terrenos planos y los resultados de tensión inducida obtenidos demuestran que la topografía influye en el funcionamiento de este elemento de protección.

Tabla 5. Máxima tensión inducida para el caso de terreno plano. Fuente: autores.

\begin{tabular}{|c|c|c|c|c|c|}
\hline \multirow{2}{*}{$\begin{array}{l}\text { Punto de } \\
\text { impacto }\end{array}$} & \multirow{2}{*}{ Ubicación del DPS } & \multicolumn{2}{|c|}{ YALUK DRAW } & \multicolumn{2}{|c|}{ FDTD 3D } \\
\hline & & $\begin{array}{l}\text { Inicio de } \\
\text { línea }(\mathrm{P} 1)\end{array}$ & $\begin{array}{c}\text { Final de } \\
\text { línea (P2) }\end{array}$ & $\begin{array}{l}\text { Inicio de } \\
\text { línea }(\mathrm{P} 1)\end{array}$ & $\begin{array}{c}\text { Final de } \\
\text { línea (P2) }\end{array}$ \\
\hline \multirow{4}{*}{ A1 } & Sin DPS & 35,5 & 8,7 & 38,7 & 13,7 \\
\hline & DPS al inicio de la línea & 35,8 & 14,2 & 30 & 20,1 \\
\hline & DPS al final de la línea & 35,6 & 17,5 & 38,6 & 24,6 \\
\hline & $\begin{array}{l}\text { la línea } \\
\text { lor y linar de }\end{array}$ & 35,9 & 25,6 & 30 & 27,4 \\
\hline \multirow{4}{*}{ A2 } & Sin DPS & 66,5 & 66,5 & 66,1 & 66,1 \\
\hline & DPS al inicio de la línea & 45,4 & 66,5 & 30,9 & 66,1 \\
\hline & $\begin{array}{l}\text { DPS al final de la línea } \\
\text { DPS al inicio y final de }\end{array}$ & 66,5 & 45,4 & 66,1 & 30,9 \\
\hline & la línea & 45,4 & 45,4 & 31,2 & 31,2 \\
\hline
\end{tabular}


Comportamiento de descargadores de sobretensión ante tensiones inducidas por rayos sobre líneas de distribución ubicadas en zona montañosa

\section{APÉNDICE}

En esta sección se presentan los resultados para terreno plano de las configuraciones analizadas en este artículo. Para esto se hace uso del software Yaluk [23][24], que obtiene el campo electromagnético mediante las ecuaciones de Master y Uman [14] y utiliza el método de acople de Agrawal para calcular las tensiones inducidas en la línea aérea. Así mismo, se presentan los resultados con el método FDTD-3D y se contrastan con los primeros. El resultado para los diferentes impactos y posiciones de los descargadores de la sección 3 se muestran en la Tabla 5.

Se puede ver en general un buen ajuste entre ambas metodologías. Las diferencias se explican porque en la simulación del Yaluk Draw, por defecto el descargador de sobretensión tiene una rama $\mathrm{R}$ - $\mathrm{L}$ en serie con el descargador que no está incluida en la simulación FDTD-3D.

\section{REFERENCIAS}

[1] M. Paolone, F. Rachidi-Haeri, and C. A. Nucci, "IEEE Guide for Improving the Lightning Performance of Electric Power Overhead Distribution Lines," IEEE Std 1410-2004 (Revision IEEE Std 1410-1997), 2010.

[2] A. Borghetti, C. A. Nucci, and M. Paolone, "An Improved Procedure for the Assessment of Overhead Line Indirect Lightning Performance and Its Comparison with the IEEE Std. 1410 Method," IEEE Trans. Power Deliv., vol. 22, no. 1, pp. 684-692, Jan. 2007. https://doi.org/10.1109/TPWRD.2006.881463.

[3] C. A. Nucci, F. Rachidi, M. V. Ianoz, and C. Mazzetti, "Lightning-induced voltages on overhead lines," IEEE Trans. Electromagn. Compat., vol. 35, no. 1, pp. 75-86, 1993. https://doi.org/10.1109/15.249398.

[4] E. Pérez and H. Torres, "Advances on modeling and experimentation of lightning induced voltages on distribution lines," 2006.

[5] M. Paolone et al., "Lightning Electromagnetic Field Coupling to Overhead Lines: Theory, Numerical Simulations, and Experimental Validation," IEEE Trans. Electromagn. Compat., vol. 51, no. 3, pp. 532-547, Aug. 2009.
https://doi.org/10.1109/TEMC.2009.2025958.

[6] E. Soto and E. Pérez, "Implementation of an analytical formulation for LEMP to assess the lightning performance of a distribution line," TecnoLógicas, vol. 21, no. 42, pp. 5162, May. 2018. https://doi.org/10.22430/22565337.778.

[7] E. Soto, E. Perez, and J. Herrera, "Electromagnetic Field Due to Lightning Striking on Top of a Cone-Shaped Mountain Using the FDTD," IEEE Trans. Electromagn. Compat., vol. 56, no. 5, pp. 1112-1120, Oct. 2014.

https://doi.org/10.1109/TEMC.2014.2301138.

[8] D. Li et al., "On Lightning Electromagnetic Field Propagation Along an Irregular Terrain," IEEE Trans. Electromagn. Compat., vol. 58, no. 1, pp. 161-171, Feb. 2016.

https://doi.org/10.1109/TEMC.2015.2483018.

[9] E. Soto, E. Perez, and C. Younes, "Influence of non-flat terrain on lightning induced voltages on distribution networks," Electr. Power Syst. Res., vol. 113, pp. 115-120, Aug. 2014.

https://doi.org/10.1016/j.epsr.2014.02.034.

[10] E. Soto, "Lightning induced voltages study on overhead distribution networks placed over non-flat at terrains," Universidad Nacional de Colombia, 2014.

[11] R. E. J. Mejía, "Lightning induced voltages on overhead lines above non-uniform and non- homogeneous ground," Universidad Nacional de Colombia, 2014.

[12] S. Yokoyama, "Distribution Surge Arrester Behavior Due to Lightning Induced Voltages," IEEE Trans. Power Deliv., vol. 1, no. 1, pp. 171-178, 1986. https://doi.org/10.1109/TPWRD.1986.430790 4.

[13] M. Paolone, C. A. Nucci, E. Petrache, and F. Rachidi, "Mitigation of Lightning-Induced Overvoltages in Medium Voltage Distribution Lines by Means of Periodical Grounding of Shielding Wires and of Surge Arresters: Modeling and Experimental Validation," IEEE Trans. Power Deliv., vol. 19, no. 1, pp. 423-431, Jan. 2004. https://doi.org/10.1109/TPWRD.2003.820196.

[14] M. A. Uman, D. K. McLain, and E. P. Krider, "The electromagnetic radiation from a finite antenna," Am. J. Phys., vol. 43, no. 1, pp. 3338, Jan. 1975. https://doi.org/10.1119/1.10027.

[15] K. S. Yee and J. S. Chen, "The finitedifference time-domain (FDTD) and the finite-volume time-domain (FVTD) methods in solving Maxwell's equations," IEEE Trans. Antennas Propag., vol. 45, no. 3, pp. 354-363, Mar. 1997. https://doi.org/10.1109/8.558651. 
Comportamiento de descargadores de sobretensión ante tensiones inducidas por rayos sobre líneas de distribución ubicadas en zona montañosa

[16] A. Z. Elsherbeni and V. Demir, The FiniteDifference Time-Domain in Electromagnetics, Har/Cdr. Institution of Engineering and Technology, 2015. https://doi.org/10.1049/SBEW514E.

[17] F. Heidler, J. M. Cvetic, and B. V. Stanic, "Calculation of lightning current parameters," IEEE Trans. Power Deliv., vol. 14, no. 2, pp. 399-404, Apr. 1999. https://doi.org/10.1109/61.754080.

[18] T. Noda and S. Yokoyama, "Thin wire representation in finite difference time domain surge simulation," IEEE Trans. Power Deliv., vol. 17, no. 3, pp. 840-847, Jul.2002.

https://doi.org/10.1109/TPWRD.2002.102281 3.

[19] G. Lin, S. Lu, and J. Liu, "Transmitting boundary for transient analysis of wave propagation in layered media formulated based on acceleration unit-impulse response," Soil Dyn. Earthq. Eng., vol. 90, no. 10, pp. 494-509, Nov. 2016. https://doi.org/10.1016/j.soildyn.2016.09.021.
[20] A. Tatematsu and T. Noda, "ThreeDimensional FDTD Calculation of LightningInduced Voltages on a Multiphase Distribution Line With the Lightning Arresters and an Overhead Shielding Wire," IEEE Trans. Electromagn. Compat., vol. 56, no. 1, pp. 159-167, Feb. 2014. https://doi.org/10.1109/TEMC.2013.2272652.

[21] SIEMENS, "Descargadores de sobretensión de media tensión 3EK4 con envolvente de Silicona," Siemens AG, Erlangen, Alemania, 2010.

[22] G. E. publicas de Medellín, "Especificaciones técnicas para descargadores de sobretensiones DPS en media tensión," 2015.

[23] E. Pérez and E. Soto, "Yaluk Draw: Software especializado para análisis del desempeño de líneas de distribución ante impacto de rayos," pp. 1-8, 2010.

[24] E. Pérez and E. Soto, "Yaluk Draw: Software especializado para análisis del desempeño de líneas de distribución ante impacto de rayos. Avances en Ingeniería Eléctrica," Av. en Ing. Eléctrica, vol. 4, no. 1, pp. 1-8, 2013. 